\title{
Metodologías de enseñanza-aprendizaje constructivista aplicadas a la educación superior
}

\author{
Constructivist teaching-learning methodologies applied to higher education
}

\section{Metodologías de enseñanza-aprendizaje}

\author{
Raquel Vera Velázquez ${ }^{(1)}$ \\ Carlos Castro Piguave ${ }^{(2)}$ \\ Ignacio Estévez Valdés ${ }^{(3)}$ \\ Kirenia Maldonado Zúñiga ${ }^{(4)}$
}

(1) Universidad Estatal del Sur de Manabí. Jipijapa. Ecuador. email: raquelita2015vera@ gmail.com. https://orcid.org/0000-0002-5071-7523

(2) Universidad Estatal del Sur de Manabí. Jipijapa. Ecuador. email: carlos.castro@ unesum.edu.ec. https://orcid.org/0000-0003-3180-2359

(3) Universidad Estatal del Sur de Manabí. Jipijapa. Ecuador. email: estevez@ unesum.edu.ec. https://orcid.org/0000-0001-8143-8466

(4) Universidad Estatal del Sur de Manabí. Jipijapa. Ecuador. email: kmzmaldonado@gmail.com. https://orcid.org/0000-0002-3764-5633

Contacto:raquelita2015vera@gmail.com

Recibido: 5-10-2020

Aprobado: 30-11-2020

\section{Resumen}

Las metodologías de enseñanza-aprendizaje utilizadas en la de educación superior pueden tener distintos enfoques, que se distinguen entre sí por la manera de aprender el estudiante. La elección de la metodología varía en función de las competencias que el docente desea obtener en sus educandos. La metodología constructivista no dispone de unas formas determinadas de enseñanza, pero sí que proporciona elementos de análisis y reflexión sobre la práctica educativa. Son muchos los autores que han aportado sus ideas y reflexiones acerca del tema. Este trabajo tiene como objetivo concretar metodologías constructivistas para el proceso de enseñanza-aprendizaje en la educación superior, con el fin de desarrollar talleres de capacitación a los docentes sobre el tema en función del protagonismo activo de los estudiantes en el desarrollo del proceso docente educativo. La investigación se llevó a cabo por medio de un estudio longitudinal descriptivo, prospectivo y cuantitativo basado en los métodos inductivo y analítico, el universo estaba compuesto por los docentes que participaron en el X Seminario Científico Metodológico de las carreras de Ingeniería Agropecuaria e Ingeniería en Computación y Redes de la Universidad Estatal del Sur de Manabí. Los resultados reflejan que los indicadores de dominio de las metodologías de enseñanza-aprendizaje constructivista de acuerdo al modelo educativo aplicado en la universidad mostraron dificultades en la aplicación adecuada, en la clase para un mejor desarrollo del proceso docente educativo.

Palabras clave: Constructivismo, enseñanzaaprendizaje, conocimiento, metodologías, proceso

\section{Summary}

The teaching-learning methodologies used in Higher Education may have different approaches, which are distinguished from each other by the way the student learns. The choice of methodology varies depending on the competences that the teacher wishes to obtain in his students. The constructivist methodology does not have specific forms of teaching, but it does provide elements for analysis and reflection on educational practice. There are many authors who have contributed their ideas and reflections on the subject. This work aims to specify constructivist methodologies for the teaching-learning process in higher education, in order to develop training workshops for teachers on 
the subject based on the active role of students in the development of the educational teaching process. The research was carried out by means of a descriptive, prospective and quantitative longitudinal study based on inductive and analytical methods, the universe was made up of teachers who participated in the $\mathrm{X}$ Scientific Methodological Seminar of the careers of Agricultural Engineering and Computer Engineering and Networks of the State University of the South of Manabí. The results reflect that the indicators of mastery of the constructivist teaching-learning methodologies according to the educational model applied in the university showed difficulties in the adequate application, in the class for a better development of the educational teaching process.

Keywords: Constructivism, teaching-learning, knowledge, methodologies, process

\section{Introducción}

La sociedad contemporánea enfrenta desafíos, que en muchos casos emanan de los avances incesantes de la ciencia y la tecnología; esto compromete a las universidades en la búsqueda de respuestas acertadas, a partir de su misión en la formación de profesionales competentes responsabilizados en la solución de problemas que demandan estos nuevos tiempos, con un espíritu científico y una voluntad transformadora del contexto económico y social.

La pedagogía constructivista muestra el camino para el cambio educativo, transformando éste en un proceso activo donde el estudiante elabora y construye sus propios conocimientos a partir de su experiencia previa y de las interacciones que establece con el maestro y con el entorno. La concepción tradicional que asumía al estudiante como un ser pasivo sin nada que aportar a la situación de aprendizaje ya no es válida, reconociendo los conocimientos y características previas con los que llega al aula, los cuales deben ser aprovechados para la construcción del nuevo conocimiento (Zapata, 2015).

Calderón, (2008), afirma que la pedagogía constructivista se centra en que la adquisición de todo conocimiento nuevo se produce a través de la movilización, por parte del sujeto de un conocimiento antiguo. El hecho de considerar que el conocimiento previo facilita el aprendizaje, es un rasgo esencial del constructivismo y que sustenta el aprendizaje significativo.
La investigación está basada en las metodologías de enseñanza-aprendizaje constructivistas aplicadas en la educación superior, lo que implica una revisión de este enfoque, tanto en sus conceptos esenciales como en la aplicación durante el proceso educativo. Estos dos aspectos tienen una interrelación, que debe ser coherente para que tenga buenos resultados. El saber didáctico no se reduce a la mera formulación de un tratado o método acerca de lo que se enseña, sino que se constituye en un campo específico del quehacer docente, que cubre toda una gama de reflexiones en torno a la relación que el profesor tiene con sus estudiantes y las condiciones en las cuales se lleva a cabo el proceso de enseñanza-aprendizaje (López, 2012).

De esta concepción dialéctica surge el estudio del constructivismo su marco teórico que sustenta una práctica pedagógica, que plantea la necesaria e ineludible relación entre la metodología y la concepción que se tiene sobre la enseñanza y el aprendizaje, así como los aspectos vinculados como es: el caso de los objetivos, los contenidos, la metodología, las técnicas y recursos, para determinar el proceso de evaluación.

El presente artículo tiene por objetivo concretar metodologías constructivistas para el proceso de enseñanza-aprendizaje en la educación superior, con el fin de desarrollar talleres de capacitación a los docentes sobre el tema en función del protagonismo activo de los estudiantes en el desarrollo del proceso docente educativo.

\section{Una mirada al constructivismo}

El origen del constructivismo se puede encontrar en las posturas de Vico y Kant planteadas en el siglo XVIII Universidad San Buenaventura (2015), e incluso mucho antes, con los griegos Araya y Andonegui (2007). El estudio aborda las teorías constructivistas como base orientadora de la metodología de enseñanza-aprendizaje, concibiendo al ser humano como un sujeto activo constructor de su realidad, por lo que logra su interacción con otras personas; posición que se complementará con los aportes de Piaget, Vygotsky y Ausubel.

En el proceso de enseñanza-aprendizaje con enfoque constructivista el profesor, al estar básicamente orientado al aprendizaje, no sólo utiliza métodos expositivos, sino trabaja en el aula con métodos participativos que motiven en los estudiantes la capacidad de pensar por sí mismos. Proyecta un problema y le da seguimiento a la exploración de los estudiantes, crea una guía para la 
indagación y promueve nuevos caminos de pensamiento. El profesor hace del aula un espacio neutro para que los estudiantes intercambien sus puntos de vista personales y los argumenten a la luz de las ideas de los demás, de modo que cada uno pueda continuar construyendo su conocimiento (Zarzar, 2004).

Para lograr los objetivos del proceso de enseñanzaaprendizaje es necesario tener en cuenta dos aspectos fundamentales del constructivismo en la pedagogía según plantea la Universidad San Buenaventura (2015), el aprendizaje es una construcción idiosincrásica: es decir, está condicionado por el conjunto de características físicas, sociales, culturales, incluso económicas y políticas del sujeto que aprende. Condicionamientos que también son válidos para quien enseña y su forma de hacerlo. Si la persona que enseña parte de la idea de que es poseedor del conocimiento que va a transmitir a los estudiantes, probablemente usará metodologías tradicionales que implican un proceso pasivo de aprendizaje, con los estudiantes en la postura de receptores del conocimiento.

En la psicología, el constructivismo se basa principalmente en la teoría de Piaget, llegando a los postulados que necesitamos, que hoy entiendan los estudiantes, el cual dice; que el desarrollo de la inteligencia es construido por el propio aprendizaje, a través de la interacción de este mismo, con el medio que los rodea.

Desde el punto de vista del constructivismo, se considera que la metodología debe reunir varias características, teniendo en cuenta el contexto: los conocimientos deben ser globales y particulares, a la vez. Esto requiere un equilibrio entre la revisión teórica de los contenidos y su aplicación particular en los contextos específicos en los que el estudiante se desenvuelve (Universidad San Buenaventura, 2015).

No es posible una aplicación a priori porque de lo contrario se vuelve imposición. Considerar los aprendizajes previos: esta es otra variable a tener en cuenta, al momento de escoger una metodología, es necesario que los docentes estén al tanto de las materias que ya se han revisado con anterioridad o, si no lo están, hacer una pequeña evaluación diagnóstica al inicio de la materia para conocer cuáles son los conocimientos que los estudiantes ya poseen.

Es importante, señalar las contribuciones de Ausubel y Vygotsky, el primero con la visión de una teoría de la asimilación y el anclaje con organizadores previos y el segundo con la visión culturalista. Ausubel enfatiza la importancia del aprendizaje significativo como elemento integrador, donde el aprendizaje se hace posible cuando se logra el anclaje con conocimientos previos. Por su parte, Vygotsky, enfatiza el aspecto cultural y la importancia de la actividad conjunta y cooperativa. Sin embargo, es conveniente considerar también los aportes de la teoría del procesamiento de la información que igualmente da una perspectiva referida a los esquemas y marcos que son codificados, reestructurados e incorporados como parte de nuestro bagaje de posibles respuestas a determinadas situaciones que plantea el medio (Zapata 2015).

Para Calderón (2008) "El constructivismo es un movimiento muy amplio que defiende la idea de que el individuo tanto en los aspectos cognitivos y afectivos, así como los simbólicos representacionales, no es un mero producto del entorno sociocultural, ni un simple resultado de disposiciones internas de carácter biológico. Es una elaboración propia que se va produciendo a lo largo de la vida por interacciones de factores básicos como: la herencia, el ambiente sociocultural, las experiencias y el lenguaje".

Las universidades latinoamericanas replantean y dinamizan sus modelos educativos, en la búsqueda de un perfeccionamiento cualitativo de los procesos formativos en concordancia, con las exigencias derivadas del encargo social. Estas instituciones están precisadas a establecer un vínculo orgánico con la sociedad, abandonando posiciones tradicionales y creando nexos con otros sectores de carácter sociopolítico que incorporen vitalidad a las posturas tradicionalmente asumidas. Zapata, Miguel (2015).

La Universidad Estatal del Sur de Manabí también afronta dichos desafíos, y por tal motivo encausa su proyección educativa hacia la implementación de un modelo educativo que se atempere a las exigencias de la sociedad ecuatoriana. El modelo de la alta casa de estudios se asume como una síntesis de la orientación epistemológica general que sirve de guía a la institución, a sus concepciones filosóficas y sociológicas, así como los principales enfoques psicopedagógicos en los cuales se basa su plataforma de acción, para dar respuesta a la demanda social. Se reflejan en las realidades de la universidad, su razón de ser, sus compromisos, su actualidad y perspectivas. 
La armonización de las nuevas tendencias paradigmicas en el contexto educativo-curricular, implica realizar una convergencia, entre la concepción filosófica del modelo, y los saberes de los enfoques holístico, constructivista, complejo, ecologista y comunicacional, pretendiendo que los currículos de formación profesional den respuesta a las diversas demandas de la humanidad y a los cambios que se operan en la ciencia y la tecnología.

Este modelo educativo implica seguir la ruta renovadora basada en el proceso de enseñanzaaprendizaje constructivista y sus metodologías, supone retomar la naturaleza misma de la educación considerando las nuevas corrientes educativas que apoyadas en una filosofía humanista, de respeto y aceptación del otro, contribuya al aprendizaje y al desarrollo integral y pleno de las personas, porque creemos que el eje del cambio social es el hombre.

\section{Materiales y métodos.}

Está investigación se llevó a cabo por medio de un estudio longitudinal descriptivo, prospectivo y cuantitativo basado en los métodos teóricos (análisis y síntesis), empíricos (técnica observación y encuestas) y métodos estadísticos para determinar la muestra y tabular los resultados del instrumento aplicado, el universo estaba compuesto por 35 docentes que participaron en el $\mathrm{X}$ Seminario Científico Metodológico de las carreras de Ingeniería Agropecuaria e Ingeniería en Computación y Redes de La Universidad Estatal del Sur de Manabí ubicada en el Campus Los Ángeles, vía Noboa Km 1 1/2 s/n, en Jipijapa con el fin de determinar los elementos del conocimiento con dificultades en las metodologías de aprendizaje constructivistas y su aplicación que inciden en el desarrollo de los procesos de enseñza-aprendizaje

Se aplicó una encuesta sobre conocimientos del proceso de enseñanza-aprendizaje constructivista y sus metodologías aplicadas en la clase. La encuesta se realizó en el $\mathrm{X}$ Seminario Científico Metodológico de preparación a los docentes, previo a la realización de los talleres del modelo educativo constructivista de la universidad, donde se comprobó el dominio de las metodologías de aprendizaje constructivistas y su aplicación metodológica en el desarrollo del proceso docenteeducativo.

Los 35 docentes formaron parte del estudio, cumpliendo con los parámetros que ameritaba el instrumento de forma anónima, el evaluador proporcionó las instrucciones del llenado y la finalidad de aplicación del cuestionario, posteriormente los datos fueron ingresados $y$ tabulados en la base de datos de Microsoft Excel, para su análisis estadístico se realizó con un intervalo de confianza del 98\%.Dentro de los aspectos éticos se garantizó el anonimato de los participantes y reserva de los datos en cada uno de los instrumentos aplicados, Tabla 1.

\begin{tabular}{|l|l|}
\hline Carreras & Docentes \\
\hline Agropecuaria & 15 \\
\hline Computación y Redes & 20 \\
\hline Total & 35 \\
\hline
\end{tabular}

Tabla 1. Docentes participantes por carreras en la investigación

\section{Resultados}

Los resultados reflejan que los indicadores de dominio de las metodologías de aprendizaje constructivista de acuerdo al modelo educativo aplicado en la universidad reflejaron dificultades en el conocimiento y utilización de la metodología más adecuada en la clase para un mejor desarrollo del proceso de enseñanza aprendizaje, presentándose los resultados en las tablas 2 y 3 .

\begin{tabular}{|l|l|l|}
\hline Carreras & Frecuencia & $\%$ \\
\hline Agropecuaria & 8 & 42,1 \\
\hline Computación y Redes & 11 & 57,8 \\
\hline Total & 19 & 100 \\
\hline \multicolumn{2}{|c|}{ Tabla 2. Dominio de las metodologías de } \\
aprendizaje constructivistas.
\end{tabular}

En las dos tablas antes mencionadas, se reflejan los resultados obtenidos en cuanto al dominio y utilización de las metodologías de enseñanzaaprendizaje donde solo el $54,2 \%$ de los sujetos demostró conocimiento de las diferentes metodologías a aplicar de acuerdo al contenido y el currículo de la asignatura y el 45,7\% demostró tener conocimientos de la aplicación de la metodología más adecuada, teniendo en cuenta las características de los estudiantes, los contenidos a impartir y los materiales a utilizar en el desarrollo del proceso de enseñanza-aprendizaje.

\begin{tabular}{|l|l|l|}
\hline Carreras & Frecuencia & $\%$ \\
\hline Agropecuaria & 7 & 43,7 \\
\hline Computación y Redes & 9 & 56,2 \\
\hline Total & 16 & 100 \\
\hline
\end{tabular}

Tabla 3. Dominio de la aplicación de las metodologías de aprendizaje constructivistas. 
La aplicación de las metodologías en el proceso de enseñanza-aprendizaje y sus elementos metodológicos a utilizar se reflejan en los resultados, como se puede apreciar en la tabla 4.La información presenta las orientaciones metodológicas que deben aplicarse para garantizar los procesos de aprendizaje interactivo, colaborativo, autónomo, participativo, conectado, contextualizado y basado en proyectos para lograr los conocimientos de aprendizajes constructivistas en el desarrollo de la clase contemporánea.

Tabla 4. Dominio de los elementos metodológicos para garantizar los procesos de aprendizajes

En el desarrollo de los procesos de enseñanzaaprendizaje interactivo, autónomo, conectado, y contextualizado solo el 51, $4 \%$ demostró los elementos que garantizan la correcta aplicación y utilización de herramienta metodológicas para el logro de estos aprendizajes.

En los procesos de aprendizajes colaborativo y participativo faltaron elementos metodológicos para demostrar cómo lograr el desarrollo de los aprendizajes, solo el 45,7\% explicó la estructura metodológica a desarrollar en cada metodología.

\section{Discusión}

Las metodologías de aprendizaje utilizadas en la educación superior pueden tener distintos enfoques, que se distinguen entre sí por la manera de aprender de la persona. La elección de la metodología varía en función de las competencias que el docente desea obtener en sus estudiantes. La metodología constructivista no dispone de unas formas determinadas de enseñanza, pero sí que proporciona elementos de análisis y reflexión sobre la práctica educativa. Son muchos los autores que han aportado sus ideas y reflexiones acerca del tema. Este trabajo trata de examinar y dar a conocer la relación entre los orígenes del Constructivismo y la actual concepción constructivista. Se desea dar un giro a los esquemas de las metodologías tradicionales hacia unas metodologías constructivistas basadas en estrategias que fomentan la participación activa del estudiante.

Se concuerdo con las investigaciones de Revelo, Collazos y Jiménez (2018) plantean que el trabajo colaborativo es un proceso en el que un individuo aprende más de lo que aprendería por sí solo, fruto de la interacción de los integrantes de un equipo, quienes saben diferenciar y contrastar sus puntos de vista, de tal manera, que llegan a generar un proceso de construcción de conocimiento.

Según el estudio realizado no solo significa agrupar a los estudiantes en equipos, sino que significa que, durante el desarrollo del proceso de aprendizaje, para aprender de manera interdependiente desarrollen tareas específicas, así como utilizar sesiones de clase que permitan la argumentación y el refuerzo del aprendizaje de cada estudiante, a través de discusiones, debates, y actividades de

\begin{tabular}{|l|l|l|}
\hline Procesos de aprendizaje & Frecuencia & $\%$ \\
\hline Interactivo & 18 & 51,4 \\
\hline Colaborativo & 16 & 45,7 \\
\hline Autónomo & 18 & 51,4 \\
\hline Participativo & 16 & 45,7 \\
\hline Conectado & 18 & 51,4 \\
\hline Contextualizado & 18 & 51,4 \\
\hline
\end{tabular}

estudio dirigidas.

La principal característica del trabajo colaborativo es que se estructura sobre la base de la formación de grupos de entre tres y seis estudiantes, donde cada miembro tiene un rol determinado y para alcanzar los objetivos es necesario interactuar y trabajar de forma coordinada. Considerando además que, tiene como objetivo final lograr que cada uno de los miembros realice con éxito sus tareas. Por su parte en el aprendizaje individual el estudiante se focaliza en conseguir sus objetivos sin tener que depender del resto de compañeros.

Barell, John (2015) indica que el aprendizaje basado en problemas (ABP o, del inglés, $\mathrm{PBL}$, problembased learning) puede definirse como un proceso de indagación que resuelve preguntas, curiosidades, dudas e incertidumbres sobre fenómenos complejos de la vida. Es un método docente basado en el estudiante como protagonista de su propio aprendizaje, donde la indagación por el alumno es una parte importante del ABP y que guiará el proceso de enseñanza-aprendizaje.

Al respecto no solo puede definirse como un proceso de indagación que resuelve preguntas, curiosidades, dudas e incertidumbres sobre fenómenos complejos de la vida, sino que el aprendizaje basado en problemas es un proceso de enseñanza-aprendizaje cíclico compuesto de muchas etapas diferentes, comenzando por hacer preguntas y adquirir conocimientos que, a su vez, llevan a más preguntas en un ciclo creciente de complejidad. 
En la metodología el Aprendizaje basado en problemas: (ABP) se coloca a los estudiantes ante situaciones problemáticas del mundo real, que les exigirán plantearse interrogantes al respecto y organizar y llevar a cabo la investigación necesaria para tener elementos que les permitan proponer una solución factible y fundamentada.

Poner en práctica esta metodología no supone sólo el ejercicio de indagación por parte de los estudiantes, sino convertirlo en datos e información útil. De acuerdo con múltiples pedagogos, las cuatro grandes ventajas observadas con el uso de esta metodología son: El desarrollo del pensamiento crítico y competencias creativas, la mejora de las habilidades de resolución de problemas, el aumento de la motivación del estudiante,la mejor capacidad de transferir conocimientos a nuevas situaciones, la utilidad del empleo de metodologías de aprendizaje activas como el aprendizaje basado en problemas (ABP) ha resultado exitoso en función de los resultados obtenidos en diferentes experiencias en diferentes ámbitos educativos.

En el ABP, el punto de partida es un problema o situación que permite al estudiante identificar necesidades para comprender mejor el problema/situación, identificar principios que sustentan el conocimiento y cumplir objetivos de aprendizaje relacionados a cada porción del programa educacional.

Esta metodología se realiza mayoritariamente a través del trabajo en grupos tutorizados y del trabajo individual autodirigido, con la finalidad de combinar la adquisición de conocimientos con el desarrollo de habilidades generales y actitudes útiles para el ámbito.

Para García, Muñoz y Basilotta (2017), el Aprendizaje Basado en Proyectos (ABP) consiste en una modalidad de enseñanza y aprendizaje centrada en tareas, un proceso compartido de negociación entre los participantes, siendo su objetivo principal la obtención de un producto final. Este método promueve el aprendizaje individual y autónomo dentro de un plan de trabajo definido por objetivos y procedimientos. Los alumnos se responsabilizan de su propio y único aprendizaje, descubren sus preferencias y estrategias en el proceso. Así mismo pueden participar en las decisiones relativas a los contenidos y a la evaluación del aprendizaje.

En correspondencia con el estudio realizado, es considerado como el trabajo que se realiza en el aula para crear servicios o productos únicos. Los estudiantes realizan una serie de tareas con un tiempo y recursos determinados, con la continua supervisión y guía del docente. En la investigación se concuerda con (García, Muñoz y Basilotta 2017).

La enseñanza basada en proyectos o tareas integradas, supone hoy la mejor garantía didáctica para una contribución eficaz al desarrollo de las competencias y al aprendizaje de los contenidos del currículo

Partiendo de un problema concreto y real, en lugar del modelo teórico y abstracto tradicional, parecen evidentes las mejoras en la capacidad de retener conocimiento por parte de los estudiantes, así como la oportunidad de desarrollar competencias complejas como el pensamiento crítico, la comunicación, la colaboración o la resolución de problemas.

UTEL (2017), plantea que el enfoque práctico de la Metodología de aprendizaje basada en casos permite el desarrollo de un alto nivel de conciencia y pensamiento crítico, a través del cual los alumnos perfilan sus habilidades directivas y se enfocan en la detección, análisis y diagnóstico de problemáticas empresariales, permitiéndoles desarrollar capacidades propias de los directivos como la toma de decisiones asertivas y un amplio criterio.

Siguiendo el estudio realizado se considera que el método de casos es una forma de enseñanza en el que los estudiantes aprenden sobre la base de experiencias y situaciones de la vida real, lo que les permite construir su propio aprendizaje en un contexto que los aproxima a su entorno. Este método se basa en la participación activa y en procesos colaborativos y democráticos de aprendizaje.

El aprendizaje basado en casos en el contexto actual de la enseñanza, donde el profesor ha dejado fundamentalmente de transmitir conocimientos para pasar a involucrarse de una forma mucho más activa en el proceso de aprendizaje, cuyo papel fundamental debe recaer en el estudiante. En este sentido la aplicación del método de estudio de casos facilita que los estudiantes asuman el papel de protagonistas activos de su propio aprendizaje, potenciando la adquisición de competencias disciplinares, genéricas y académicas y discusión de la situación reflejada en el caso. 
Castro (2020), en su artículo ¿de qué trata Aprendizaje interactivo? plantea que es un aprendizaje dinámico, asociado al movimiento que se caracteriza por proporcionar actividades motivadoras que impulsan a la persona para que se mantenga activa y profundice en el conocimiento y que se pueden incluir diversas herramientas que ayuden a conseguir los objetivos marcados.

En correspondencia con lo planteado por Castro (2020), sobre el aprendizaje interactivo consideramos que para aplicar el aprendizaje interactivo deben tener conocimiento del medio y sujetos de interacción, se debe asegurar de la eficacia de los canales para la interactividad entre los estudiantes y el ambiente, así como el contenido sea pertinente con los objetivos de las asignaturas y los procesos pedagógicos : Lluvia de ideas, elaboración de tablas, cuadros comparativos, resolución de problemas, estudio de casos, foro de presentación, preparación de ensayos, presentación de informes y presentación de informe digital.

Escobedo, Pimentel y Cruz (2020), plantean que el aprendizaje autónomo, es un término actual creado con la finalidad de dar respuesta a las necesidades del trabajo moderno en donde los tiempos son escasos para dedicar al aprendizaje, es un término innovador toda vez que se pretende dar respuesta a las problemáticas que presenta el sistema a distancia, así mismo el aprendizaje autónomo se encuentra relacionado con los avances tecnológicos que día a día aumentan de forma inmensurable. El aprendizaje autónomo permite a los sujetos de disponer y organizar sus tiempos de conformidad a sus necesidades.

Siguiendo lo planteado por Escobedo, Pimentel y Cruz (2020) para lograr un buen desarrollo del aprendizaje autónomo se tiene que tener presente algunos elementos del aprendizaje, análisis de información, elaboración de síntesis, presentación de organizadores gráficos, lectura y análisis de material, lectura de documentos, análisis de información estadística, manejo de bases de datos y acervos bibliográficos, elaboración de artículos científicos y lectura de bibliografía especializada.

El aprendizaje autónomo no es solo la capacidad de aprender por uno mismo, sin necesidad de alguien más, si no que esta actuación debe estar encaminada a identificar los niveles de autonomía que exigen los contenidos y los procesos y a conocer el nivel de motivación del estudiante hacia el aprendizaje autónomo y su preparación.
Para un buen desarrollo del aprendizaje autónomo tiene que tener presente algunos elementos del aprendizaje, análisis de información, elaboración de síntesis, presentación de organizadores gráficos, lectura y análisis de material, lectura de documentos, análisis de información estadística, manejo de bases de datos y acervos bibliográficos, elaboración de artículos científicos y lectura de bibliografía especializada.

Siguiendo lo planteado por Moreno, (1993) el aprendizaje participativo no solo como una metodología que logra que tus clases sean activas y no exista el aburrimiento, si no que la metodología exige asegurar que existan condiciones de participación y que la participación sea real y activa. Evaluar la participación individual en la concepción del grupo de trabajo o aprendizaje.

Teniendo en cuenta actividades participativas como: lluvia de ideas, construcción de documentos audiovisuales, diálogo informativo, prácticas de laboratorio, campo, trabajos de observación documentación y experimentación dirigida, prácticas pre profesionales, visita guiada de campo, presentación de proyectos de integración de saberes, análisis de información, presentación de informes contextualizados, presentación del material elaborado, estudio de casos y preparación de documentales.

En correspondencia con lo planteado por Miró (2016), sobre el aprendizaje basado en competencias con un enfoque de la educación que se centra en la demostración de los resultados de aprendizaje deseados como el centro del proceso de aprendizaje del estudiante. Se refiere principalmente a la progresión del estudiante a través de planes de estudio a su propio ritmo, profundidad, etc. Como han demostrado las competencias, los estudiantes continúan progresando.

Coincidiendo con lo referido anteriormente sobre el aprendizaje basado en competencias, se plantea que, todo aprendizaje tiene como objetivo la adquisición de conocimiento, el desarrollo de habilidades y la solidificación de hábitos de trabajo. El aprendizaje basado en competencias representa un conjunto de estrategias para lograr esta finalidad y la evaluación es un factor importante en la aplicación de esta metodología consiste en desarrollar las competencias genéricas o transversales necesarias y las competencias específicas (propias de cada profesión), con el propósito de formar a las personas sobre los conocimientos científicos y técnicos, su capacidad de aplicarlos en contextos 
diversos y complejos, integrándolos con sus propias actitudes y valores en un modo propio de actuar desde el punto de vista personal y profesional.

\section{Conclusiones}

La percepción que tienen los docentes en cuanto al proceso de enseñanza-aprendizaje constructivista y su metodología es positiva, lo que resulta importante la utilización de herramientas que conlleven a estrategias metodológicas en la aplicación del aprendizaje basado en problemas, proyectos, estudio de casos y trabajo autónomo para lograr mejores resultados en el desarrollo del proceso de enseñanza-aprendizaje.

Se identificaron las dificultades de los docentes en el dominio y aplicación de las metodologías de enseñanza-aprendizaje constructivista según las exigencias del modelo educativo de la educación superior, con el fin de desarrollar talleres de capacitación sobre las teorías de aprendizaje constructivista y sus metodologías para lograr un mejor desarrollo del proceso de enseñanzaaprendizaje. Evidenciado en el 51,4\% que demostró los elementos que garantizan la correcta aplicación y utilización de herramienta metodológicas para el logro de estos aprendizajes. En los procesos de aprendizajes colaborativo y participativo faltaron elementos metodológicos para demostrar cómo lograr el desarrollo de los aprendizajes, solo el 45,7 $\%$ demostró la estructura metodológica a desarrollar en cada metodología.

En el estudio se concretaron metodologías para el desarrollo del proceso de enseñanza -aprendizaje en la educación superior con un enfoque constructivista, en función del protagonismo activo de los estudiantes en actividades teórico prácticas.

\section{Bibliografía}

Araya, V., Alfaro, M. y Andonegui M. (2007). Constructivismo: orígenes y perspectivas. Revista Laurus. 13, (24).76-92. Recuperado de: https://www.redalyc.org/pdf/761/7611148500 4.pdf

Barell, J. (2015). El aprendizaje basado en problemas: Un enfoque investigativo (Marcelo Pérez Rivas, Argentina. Ed. Manantial.

Castro, María. (2020). ¿De qué trata el aprendizaje interactivo? Universidad de Salamanca.
Calderón, R. (2008)."Constructivismo y aprendizajes significativos". Recuperado de: https://www.monografias.com/trabajos7/apren d/aprend.shtml

Escobedo, R., Pimentel, A. y Cruz, C. (2020): El aprendizaje autónomo en el sistema a distancia”, Revista de Desarrollo Sustentable, Negocios, Emprendimiento y Educación RILCO DS, 7 Recuperado de: http://hdl.handle.net/20.500.11763/rilcoDS07a prendizaje-autonomo

Farnós, J. (2016). ¿Qué es el aprendizaje basado en competencias? Recuperado de: https://juandomingofarnos.wordpress.com/201 6/04/18/que-es-el-aprendizaje-basado-encompetencias/

García-Varcálcel Muñoz-Repiso, A. y Basilotta Gómez-Pablos, V. (2017). Aprendizaje basado en proyectos (ABP): evaluación desde la perspectiva de alumnos de Educación Primaria. Revista de Investigación Educativa, 35(1), 113-131, Recuperado de: http://dx.doi.org/10.6018/rie.35.1.246811Lópe

$\underline{\mathrm{Z}}$

Pérez, R. (2012). Idea de Constructivismo. Recuperado http://www.periodismo.uchile.cl/cursos/ psicologia/constructivismo.pdf

Moreno, S. (1993).Guía de aprendizaje participativo. Ed. Trillas, México

Sánchez, O., Collazos, C. y Jiménez, J. (2018). El trabajo colaborativo como estrategia didáctica para la enseñanza/aprendizaje de la programación: una revisión sistemática de literatura. Revista Tecnológicas, 21, (41), 115134. Recuperado de: http://www.scielo.org.co/pdf/teclo/v21n41/v2 $\underline{1 \mathrm{n} 41 \mathrm{a} 08 . \mathrm{pdf}}$

Universidad San Buenaventura (2015) Las corrientes constructivistas y los modelos autoestructurantes. En: N.N., Los modelos pedagógicos, Bogotá: Universidad San Buenaventura.143-185.

Universidad Tecnológica Latinoamericana, (UTEL) (2017). Metodología de Aprendizaje Basada en Casos,UTEL-Blog. Recuperado de: https://www.utel.edu.mx/blog/menuprofesional/facultad-deestudios-de- 
posgrado/metodologia-de-aprendizaje-basada$\underline{\text { en-casos }}$

Zapata, M. (2015). Las corrientes constructivistas y los modelos autoestructurantes. En: N.N., Los modelos pedagógicos, Bogotá: Universidad San Buenaventura.143-185.

Zarzar Charur, C (2004). Habilidades básicas para la docencia. Edit. Patria, México, 147. 